\title{
Frontières
}

\section{L'expérience du vieillissement chez des femmes et des hommes vivant avec le VIH : un vécu à l'intersection du genre, de l'orientation sexuelle et du parcours relié au VIH}

\section{Isabelle Wallach}

Volume 25, numéro 1, automne 2012

Le vieillissement et sa diversité

URI : https://id.erudit.org/iderudit/1018233ar

DOI : https://doi.org/10.7202/1018233ar

Aller au sommaire du numéro

Éditeur(s)

Université du Québec à Montréal

ISSN

1916-0976 (numérique)

Découvrir la revue

Citer cet article

Wallach, I. (2012). L'expérience du vieillissement chez des femmes et des hommes vivant avec le VIH : un vécu à l'intersection du genre, de l'orientation sexuelle et du parcours relié au VIH. Frontières, 25(1), 105-126.

https://doi.org/10.7202/1018233ar
Résumé de l'article

Les personnes vivant avec le VIH sont considérées comme « âgées " dès l'âge de 50 ans en raison du vieillissement physique prématuré qu'elles subissent. Compte tenu de l'âgisme qui prévaut dans les sociétés occidentales, on peut se demander comment les personnes vivant avec le VIH de 50 ans et plus (PVVIH50+) perçoivent leur vieillissement physique. S'appuyant sur des entrevues réalisées avec 38 PVVIH50+, cet article se propose d'explorer leur expérience du vieillissement à travers trois dimensions, à savoir le vieillissement physique, le sentiment de vieillir et le rapport au vieillissement. L'analyse des données révèle que le vieillissement revêt à la fois un caractère négatif et positif pour les PVVIH50+. Si, comme la population générale, elles tentent de s'en distancier et peinent parfois à l'accepter, le parcours relié au VIH peut en revanche teinter le vieillissement d'une valeur positive, puisque vieillir signifie être encore en vie. 


\section{L'EXPÉRIENCE DU VIEILLISSEMENT CHEZ DES FEMMES ET DES HOMMES VIVANT AVEC LE VIH: UN VÉCU À L'INTERSECTION
DU GENRE, DE L'ORIENTATION
SEXUELLE ET DU PARCOURS
RELIÉ AU VIH}

Isabelle Wallach Professeure, Département de sexologie, Université du Québec à Montréal

\section{RÉSUMÉ}

Les personnes vivant avec le VIH sont considérées comme «âgées» dès l'âge de 50 ans en raison du vieillissement physique prématuré qu'elles subissent. Compte tenu de I'âgisme qui prévaut dans les sociétés occidentales, on peut se demander comment les personnes vivant avec le VIH de 50 ans et plus (PVVIH50+) perçoivent leur vieillissement physique. S'appuyant sur des entrevues réalisées avec 38 PVVIH50+, cet article se propose d'explorer leur expérience du vieillissement à travers trois dimensions, à savoir le vieillissement physique, le sentiment de vieillir et le rapport au vieillissement. L'analyse des données révèle que le vieillissement revêt à la fois un caractère négatif et positif pour les PVVIH50+. Si, comme la population générale, elles tentent de s'en distancier et peinent parfois à l'accepter, le parcours relié au VIH peut en revanche teinter le vieillissement d'une valeur positive, puisque vieillir signifie être encore en vie. 
Individuals living with HIV are considered "elderly" as early as age 50, due to the premature physical ageing they undergo. Given the widespread ageism in western societies, it can be wondered how people living with HIV aged 50 and over (PLHIV50+) perceive their own physical ageing. In this article, based on 38 in-depth interviews conducted with PLHIV50+, the experience of ageing will be explored through three dimensions, namely the physical ageing, the feeling of ageing, and the attitudes toward ageing. Data analysis shows that perceptions about ageing are both negative and positive among PLHIV50+. Indeed, as it is observed in the general population, PLHIV50+ try to distance themselves from age and have sometimes trouble accepting it, but their experience of HIV can however bring a positive value to age, as growing old means being still alive.

MOTS-CLÉS: vieillissement - VIH - corps - genre - orientation sexuelle - âgisme

KEYWORDS: ageing - HIV - body - gender - sexual orientation - ageism

\section{INTRODUCTION}

Bien que l'infection à VIH reste perçue comme une maladie touchant principalement les jeunes, une autre réalité se manifeste dans les pays occidentaux et notamment au Canada. Ainsi, en dépit des préjugés, la population vivant avec le VIH vieillit. Ce phénomène s'explique tout d'abord par l'allongement de l'espérance de vie des personnes infectées grâce à l'avènement de thérapies antirétrovirales hautement efficaces qui ont transformé le VIH en une maladie chronique. Par ailleurs, l'augmentation de la proportion de personnes âgées vivant avec le VIH trouve également son origine dans I'accroissement continu de la proportion des personnes qui contractent ce virus à l'âge de 50 ans ou plus. Précisons ici que dans le contexte du 
VIH, on parlera généralement de personnes âgées à propos de personnes de 50 ans et plus en raison des catégories d'âge utilisées historiquement par le Center Disease Control aux États-Unis pour assurer la surveillance de l'évolution de l'épidémie (Emlet et al., 2010), usage qui s'est généralisé dans la littérature scientifique. En outre, le choix de cet âge précoce pour désigner la catégorie des personnes âgées dans le contexte du VIH peut se justifier par le vieillissement physique prématuré que subissent les personnes vivant avec le VIH (PVVIH) (Desquilbet et al., 2007; Effros et al., 2008)1. Selon la littérature scientifique, le vieillissement accéléré des PVVIH se traduit par une détérioration de la santé et l'apparition de comorbidités, dont les plus courantes sont les troubles articulaires, les troubles osseux, les troubles métaboliques, les maladies cardiaques, les cancers, I'hypertension et les troubles cognitifs (Brennan et al., 2009).

Alors que la vie avec le VIH confronte les individus à de multiples problématiques tant sur les plans psychologique, social, que médical, vieillir avec cette pathologie représente un défi supplémentaire, du fait des nombreux ajustements physiques et sociaux auxquels doit faire l'individu. La littérature traite des nombreuses difficultés psychosociales auxquelles sont confrontées les PVVIH50+ du fait de leur positionnement à l'intersection du VIH et du vieillissement (Brennan et al., 2009; Power et al., 2010; Emlet et al. 2004). Néanmoins l'expérience du vieillissement des PVVIH50+, et en particulier de leur vieillissement physique, demeure totalement inexplorée.

Les recherches en gérontologie sociale et critique montrent la complexité du rapport au vieillissement au sein de la population générale du fait de l'âgisme prégnant au sein de notre société. Introduit par Butler en 1969, le concept d'âgisme désigne le «processus selon lequel une personne est stéréotypée et discriminée en raison de son âge» (Lagacé, 2010, p. 2). Ce phénomène se perçoit notamment à travers les images négatives associées à la vieillesse, telles que le déclin et la dépendance, mais aussi dans les images positives et la valorisation d'un vieillissement «réussi» et actif, qui agissent comme une injonction à lutter contre les manifestations du vieillissement et exclut les personnes ne pouvant se conformer à ces modèles (Katz, 2009; voir dans ce numéro l'article de Burns et al.). Le corps joue souvent un rôle central dans les expériences de discrimination et de marginalisation 
reliées à l'âge (Ward et Holland, 2011; Featherstone, 1995) contribuant à en faire un des lieux fondamentaux où se joue et se construit l'expérience du vieillissement. De nombreux travaux ont par ailleurs montré que la progression du vieillissement est souvent évaluée par les individus à l'aune des changements corporels et de la détérioration des aptitudes physiques qu'ils ressentent (Balard, 2011; Hummel, 2011). La littérature met cependant en lumière les différences pouvant exister dans le vécu et l'interprétation des signes physiques de vieillissement en fonction du genre et de l'orientation sexuelle des personnes âgées (Hurd 1999; Hurd Clarke et Korotchenko, 2011; Ward et Holland, 2011; Slevin et Linneman, 2009).

Partant du constat de la difficile acceptation du vieillissement dans notre société et de la centralité du corps dans la construction de l'expérience du vieillissement, la question que nous nous proposons de traiter est celle de savoir comment est vécu le phénomène du vieillissement par des personnes atteintes d'une pathologie ayant été considérée dans les premières années de son apparition comme mortelle ${ }^{2}$ et qui parallèlement provoque un vieillissement prématuré. Cet article vise donc à cerner en quoi le fait de vivre avec le VIH influe sur l'expérience et le vécu du vieillissement, notamment dans ses dimensions physiques, tout en portant attention aux spécificités de ce vécu au regard des positionnements sociaux des individus en termes de genre et d'orientation sexuelle. Après avoir décrit la méthodologie sur laquelle s'appuie notre recherche, nous nous intéresserons à l'expérience du vieillissement physique des PVVIH50+, à son impact sur leur sentiment de vieillir pour ensuite nous pencher sur leur rapport au vieillissement.

\section{MÉTHODOLOGIE}

Les données présentées dans cet article sont issues d'un projet de recherche portant sur la vie personnelle et sociale des personnes vivant avec le VIH de 50 ans et plus (PVVIH50+). Ce projet s'inscrit dans une approche qualitative qui met l'accent sur l'analyse des significations et vise une compréhension approfondie de l'expérience des individus (Denzin et Lincoln, 1994) relatifs à l'intersection du VIH et du vieillissement. 


\section{Recrutement et échantillon}

Les participants ont été recrutés au sein d'une des plus importantes cliniques médicales spécialisées dans le VIH/sida au Canada, située à Montréal au Québec. L'unique critère d'inclusion était que les personnes soient âgées de 50 ans ou plus et vivent avec le VIH. L'échantillon a été diversifié selon divers critères, à savoir le genre, l'orientation sexuelle, l'âge, I'ancienneté du diagnostic. Il inclut également des participants de minorités ethniques et des utilisateurs de drogues par injection (UDI) (anciens ou actifs) afin de refléter la diversité des populations âgées affectées par le VIH. L'échantillon se compose de 38 PVVIH50+, âgées de 50 à 73 ans. Vingt-trois sont dans la cinquantaine, douze dans la soixantaine et trois sont des septuagénaires. L'échantillon inclut 12 hommes ayant des rapports sexuels avec des hommes (HARSAH), 14 femmes hétérosexuelles et 12 hommes hétérosexuels, parmi lesquels 8 font partie d'une minorité ethnique (six afro-caribéens, 1 latino et 1 asiatique) et huit sont des UDI. Concernant la situation socioéconomique des participants, 13 sont en situation d'emploi, 13 sont en situation de non-emploi et 12 sont retraités. En raison de leur statut professionnel, la majorité des participants sont en situation de précarité économique. Ainsi, près des deux tiers des participants (24/38) ont des revenus annuels inférieurs à $20000 \$$, huit ont des revenus annuels se situant se situent entre 20000 et 50000 \$ et seulement six participants gagnent plus de 50000 \$ par an. Précisions que le recrutement des participants a été arrêté quand la saturation des données a été atteinte.

\section{- Collecte des données}

La recherche s'appuie sur des entrevues individuelles semi-dirigées approfondies d'une durée moyenne de deux heures trente, qui ont été conduites à Montréal, dans les locaux administratifs de la clinique médicale où a eu lieu le recrutement ou, exceptionnellement, au domicile des participants. Ces entrevues ont été réalisées soit par la chercheure principale soit par un des deux assistants formés à cet effet. Tous les participants ont été informés des objectifs de la recherche et invités à signer un formulaire de consentement avant le début de l'entrevue, qui les informait de leur droit à ne pas répondre aux questions et à se retirer en tout temps du projet, ainsi que sur les procédures 
relatives au respect de la confidentialité et de l'anonymat. Les entrevues portaient sur l'expérience subjective du VIH et du vieillissement et son impact sur les différentes dimensions de la vie personnelle et sociale de l'individu. Une compensation symbolique de $40 \$$ a été remise à tous les participants à la fin de l'entrevue.

\section{Analyse des données}

Toutes les entrevues ont été intégralement retranscrites. L'analyse des données s'est basée sur l'approche de la théorisation ancrée (Strauss et Corbin, 1998). Elle a été réalisée avec l'aide du logiciel QDA Miner. La lecture des premières entrevues nous a permis d'établir une première liste de catégories et de codes à partir des thématiques récurrentes identifiées dans les discours mais aussi de thèmes issus de la grille d'entrevue ou de la littérature. Un travail de relecture régulière du matériel associé à chaque code a permis de raffiner nos codes et d'identifier des catégories d'analyse. Nous avons ainsi progressivement défini des sous-catégories de la catégorie «expérience du vieillissement», telles que vieillissement physique, sentiment de vieillir, rapport au vieillissement (acceptation/refus), inquiétudes reliées au vieillissement, impact du vieillissement. L'étape suivante a consisté à comparer les expériences des différents sous-groupes de populations inclus dans notre échantillon afin de mieux saisir les variations des expériences du vieillissement en fonction de variables telles que le genre et l'orientation sexuelle.

Les résultats, présentés dans les sections suivantes, s'organiseront autour des trois grands thèmes ayant émergé de l'analyse, à savoir l'expérience du vieillissement physique, le sentiment de vieillir et le rapport au vieillissement.

\section{LE VIEILLISSEMENT PHYSIQUE}

La quasi-totalité des participants perçoit des signes d'un vieillissement physique, lesquels peuvent revêtir différentes formes. Les problèmes de santé ne sont que rarement interprétés comme des marqueurs du vieillissement malgré le fait que la grande majorité des répondants en soient atteints. En réalité, l'inscription du vieillissement dans le corps 
semble davantage être expérimentée à travers les changements de l'apparence que la détérioration de la santé. Plus des deux tiers des participants considèrent les changements corporels comme le principal signe de leur vieillissement. Les changements corporels associés au vieillissement les plus fréquemment cités concernent la transformation de la chevelure (perte, grisonnement, blanchiment), l'apparition de rides sur le visage, le relâchement des tissus, la perte musculaire ou la prise de poids. Par ailleurs, ces changements de l'apparence semblent davantage interprétés comme des signes de vieillissement par les HARSAH et les femmes que par les hommes hétérosexuels.

Le second signe de vieillissement physique, évoqué par environ la moitié des participants, a trait à la baisse de l'énergie ou de la force physique, qui se manifeste par la perte de leurs aptitudes physiques, la baisse de leur résistance ou une plus grande fatigue ressentie en accomplissant des activités auparavant réalisées sans effort. À l'inverse des transformations de l'apparence, on peut noter que la diminution de la forme physique est plus fréquemment considérée comme un marqueur de vieillissement par les hommes hétérosexuels que par les HARSAH et les femmes.

Enfin, les troubles de santé comme les maladies et les douleurs sont associés à un vieillissement physique par seulement une dizaine de participants. De même, les pertes sensorielles (vue, ouïe) et les troubles cognitifs, qui affectent plus des deux tiers des participants, ainsi que les changements physiologiques (ménopause, troubles érectiles) qui touchent la moitié des participants, sont rarement cités comme des signes de vieillissement physique.

Une spécificité qui se dégage par ailleurs des discours des participants a trait au caractère prématuré de leur vieillissement physique. Plusieurs répondants s'interrogent ainsi sur le caractère anormalement précoce des signes de vieillissement qu'ils observent dans leur corps, se demandant s'ils ne peuvent être attribués à l'infection à VIH. Un répondant âgé de 60 ans raconte que ses interrogations sont nées du constat d'un vieillissement accéléré des traits de son visage comparativement aux autres hommes de sa famille.

Mais là, tout le temps j'essaie de comprendre, je dis: «Mais comment ça je vieillis plus que mon frère qui a 67 ? ». Et puis c'est là, j'ai dit: «Ah! ça se pourrait c'est la maladie qui me vieillit 
plus. ». (...) J'ai regardé des photos. Finalement j'ai dit: «Mais où est mon père? Où est moi ? Est-ce que ça, c'est ma photo ou la photo de mon père. ». C'est après j'ai réalisé, j'ai dit: «Mais non, ce n'est pas mon père ça, ça, c'est moi! » $(\mathrm{HH} 60)^{3}$

Au regard des registres de changements corporels reliés au vieillissement mentionnés par les participants (apparence, énergie et santé), il appert que les PVVIH50+ perçoivent effectivement les signes d'un vieillissement physique prématuré. Les données recueillies mettent cependant en lumière un écart existant entre la définition médicale du vieillissement prématuré associé au VIH, basée sur la présence de comorbidités, et l'expérience subjective du vieillissement physique des PVVIH50+ qui s'enracine principalement dans I'observation de changements au niveau de l'apparence et de l'énergie.

La seconde question qui semble intéressante à soulever est celle de savoir si ces signes physiques précoces de vieillissement contribuent pour autant à forger un sentiment de vieillir chez les PVVIH50+, surtout si I'on considère que même les populations plus âgées marquent une tendance généralisée à vouloir se distancier de l'identité de personne âgée étant donné les préjugés négatifs entourant le vieillissement dans notre société.

\section{LE SENTIMENT DE VIEILLIR}

Les discours des PVVIH50+ révèlent la relation complexe existant entre les signes physiques du vieillissement et le sentiment de vieillir. Deux postures majeures émergent ainsi de l'analyse: I'ambivalence relative au sentiment de vieillir et la reconnaissance du sentiment de vieillir ou d'avoir vieilli.

\section{- L'ambivalence relative au sentiment de vieillir}

La majorité des participants se montrent ambivalents au regard du sentiment de vieillir, affirmant, d'une part, ressentir des signes de vieillissement et, d'autre part, ne pas se sentir vieux ou se sentir encore jeune. Ce sentiment ambivalent se manifeste à travers trois modes de relativisation de leurs signes physiques du vieillissement: I'expérience 
corporelle contradictoire de l'individu, le regard des autres et la comparaison de son propre état physique à celui des autres.

On peut tout d'abord relever une tendance de nombreux participants à relativiser leur vieillissement physique en prenant appui sur d'autres signes physiques perçus comme représentatifs du maintien de leur jeunesse. Ces signes corporels de jeunesse peuvent s'inscrire dans le même registre que les signes de vieillissement repérés ou dans des registres différents (apparence, énergie/force ou santé). Ainsi, plusieurs participants considèrent leur forme physique et leur aptitude à conserver le même rythme de vie ou à accomplir certaines activités comme un signe de jeunesse et l'utilisent pour prendre de la distance par rapport au vieillissement de leur apparence, à l'instar de cette participante. «Sur des photos, je me regarde, oui, là tu te vois vieille. Mais je veux dire je suis tellement active puis game pour n'importe quoi! » (F62)

Dans certains cas, c'est l'apparence jeune de certaines parties de leur corps qui sert à nuancer le vieillissement d'autres zones corporelles, comme dans cet extrait d'entrevue: «Ce n'est pas parce que j'ai des cheveux blancs que je suis à moitié mort. Parce que physiquement, moi l'été je me promène en T-shirt, t'sais je suis développé [musculairement].» (HH65)

Plusieurs participants ont également fait référence au décalage existant entre leur âge chronologique et l'âge moins élevé que leur attribue des personnes de leur entourage. Le regard des autres, qui renvoie une image de personne plus jeune, semble ainsi largement contribuer à la relativisation du vieillissement physique, comme l'exprime cette femme. «Je me sens pas vieillir. Premièrement on me donne toujours plus jeune que mon âge. » (F60)

Enfin, le sentiment de rester jeune malgré la perception de signes physiques de vieillissement peut prendre appui sur la comparaison de son état physique avec celui de personnes du même âge ou plus âgées. Il peut notamment provenir du décalage entre les représentations des participants relatives à ce qu'ils considèrent comme des attributs de la vieillesse et leurs propres signes de vieillissement. Une participante explique ainsi se sentir encore jeune malgré les signes de 
vieillissement affectant son apparence car elle conserve encore toute sa forme physique, à la différence de ses parents plus âgés.

Comme mes parents, quand ils faisaient des voyages avec des gens, des groupes de personnes âgées ils s'appelaient, entre eux, les «Tamalou, » t'as mal où? On rit beaucoup de ça, mais ils sont rendus là. On va tous y arriver, là. Mais moi, pour le moment, non. À part mes cheveux blancs et quelques rides, peut-être (rires), mais non. (F53)

Pour finir, il convient de souligner qu'il n'est pas toujours aisé de distinguer, dans les discours des participants, ce qui relève d'une ambivalence à l'endroit du sentiment de vieillir en raison de signes physiques de jeunesse qui viennent contredire l'expérience d'un vieillissement corporel et ce qui s'enracine dans une intériorisation de l'âgisme prégnant au sein des sociétés occidentales qui conduit les participants à vouloir se distancier de l'identité stigmatisée de la personne âgée. Cette seconde situation apparaît avec évidence lorsque les participants nient au début de l'entrevue avoir ressenti des signes d'affaiblissement physique alors qu'ils les évoquent plus tardivement. Un même participant affirme ainsi dans la première partie de l'entrevue: «Je ne sens pas que j'ai 65 ans. Je suis encore assez actif. Je fais du vélo. Je suis venu ici à pied, ça m'a pas du tout fatigué», pour ensuite reconnaître: «On perd un peu de force, on n'a plus les mêmes capacités à 65 ans. Comme aller en vélo, avant je faisais des longues rides [promenades], maintenant, oups, je raccourcis, je sens que je suis plus essoufflé». (HARSAH65)

\section{La reconnaissance du sentiment de vieillir}

La posture de la reconnaissance du sentiment de vieillir s'est avérée beaucoup moins répandue chez les participants puisqu'elle ne concerne qu'une douzaine d'entre eux. Dans la plupart des cas, ce sentiment se fonde sur le constat d'une perte des aptitudes physiques, d'une détérioration de la santé ou d'une grande fatigue qui se répercute sur la vie quotidienne en empêchant les personnes de poursuivre les activités qu'elles avaient I'habitude de réaliser. Un participant explique ainsi: «Je vais avoir 53. Je suis vieux! Parce que quand j'étais jeune, j'avais des forces. Maintenant, c'est pas la même force. Moi, je levais des 
poids 100 livres, tu comprends? Maintenant, j'ai besoin de quelqu'un pour m'aider. 》 (HH52)

Plus rarement, il est apparu que le seul changement de l'apparence physique pouvait entraîner le sentiment d'être devenu vieux, lorsque ces changements corporels avaient des répercussions sur les relations avec autrui ou bien sur l'estime de soi, dans le cas de personnes ayant accordée une grande importance à l'apparence corporelle durant leur parcours de vie. Le sentiment de vieillir est d'autant plus fort lorsque ce sont les autres qui renvoient cette image de personne âgée et que I'individu en ressent effectivement les conséquences dans sa vie sociale ou intime. Deux participants ont ainsi évoqué les conséquences néfastes du changement de leur apparence qui contribue à la construction de leur sentiment d'avoir vieilli.

C'est sûr qu'on a ... je le sais en vieillissant, on vieillit, on a les traits physiques qui se dégradent, surtout rendue à 56 ans, ça commence ... Déjà t'sais moi j'étais ... j'ai toujours été un peu coquette, puis aimer plaire. Mais là, tu te sens de plus en plus isolée, puis les gens te parlent de moins en moins, puis ils ne t'abordent pas comme avant. (F56)

Enfin, on peut remarquer que plusieurs participants ayant le sentiment d'avoir vieilli, rapportent néanmoins éprouver un sentiment de décalage entre leur vieillissement physique, perçu à travers le changement de leur apparence physique ou la détérioration de leur santé, et leur sentiment intime d'être encore jeune de «cœur» ou de «mentalité».

Non, je suis vieux! Parce que quand j'étais jeune, j'avais des, des forces. Maintenant, c'est pas la même force, tu comprends? Maintenant, j'ai besoin de quelqu'un pour m'aider. (...) Les plis de ma figure, les cheveux sont blanchis. De plus en plus. (...) Peut-être le visage te fait remarquer l'âge, mais, le cœur ne vieillit jamais. Dans le cœur, tu es toujours jeune. (HH52)

L'ensemble des données montrent par conséquent que les PVVIH50+ sont confrontées, souvent dès la cinquantaine, à l'expérience d'un vieillissement physique et à l'ambigüité relative au sentiment de vieillir. $\mathrm{Au}$ regard de la dévalorisation du vieillissement qui traverse notre société, on pourrait s'attendre à ce que cette population exposée précocement au vieillissement nourrisse un sentiment négatif à son 
endroit. Nous allons donc à présent nous pencher sur la question du rapport des PVVIH50+ au vieillissement et constater que le VIH peut avoir une influence à la fois positive et négative sur ce rapport.

\section{- LE RAPPORT AU VIEILLISSEMENT}

Alors que la moitié des participants affirme bien accepter le fait de vieillir et la perspective de la vieillesse, l'autre moitié émet des réserves quant à son acceptation du vieillissement actuel ou de la vieillesse à venir. Le rapport au vieillissement s'avère largement influencé par le parcours relié au VIH mais aussi par l'orientation sexuelle et le genre des participants.

\section{- Un rapport difficile au vieillissement}

La quasi totalité des participants ayant fait part des raisons de leur vécu négatif actuel du vieillissement, l'attribuent à la difficile acceptation de la transformation de leur apparence physique, les HARSAH étant les plus affectés par ce phénomène. Un nombre peu élevé et égal d'hommes hétérosexuels et de femmes associent, en revanche, leur difficile acceptation du vieillissement au changement de leur apparence physique. Les femmes se révèlent toutefois plus nombreuses à vivre difficilement le changement de leur apparence, quand bien même ce vécu n'a pas d'incidence directe sur leur acceptation du vieillissement. De ce fait, il semblerait que le vécu négatif du vieillissement soit davantage relié à l'intériorisation des normes relatives à l'identité de genre ou à I'orientation sexuelle et aux idéaux corporels qui leur sont associés qu'à l'infection à VIH.

Les participants HARSAH évoquent ainsi leur difficile acceptation du vieillissement en raison de la perte de leur pouvoir de séduction qui en découle, compte tenu du culte de la jeunesse, de la beauté et des corps parfaits qui prévaut au sein de la communauté gaie, comme le montrent ces propos: «Sans paraître prétentieux, mais disons que j'avais la chance d'être assez beau bonhomme, dans la vingtaine. Et puis j'étais très populaire. Et je dois vous dire, la part du vieillissement qui a été le 
plus pénible c'était de voir que doucement, ce pouvoir de séduction là s'effritait. 》 (HARSAH53)

Plusieurs femmes expriment quant à elles un vécu douloureux face au changement de leur silhouette associé au vieillissement et à l'apparition de rides sur leur visage, bien que, à la différence des HARSAH, la plupart insistent sur la nécessité d'accepter de tels changements corporels.

Puis le vieillissement, bien là, comme j'ai maigri beaucoup, regarde. Yark! C'est dégueulasse là, c'est pas beau. Tu vois une vieille qui se promène avec des shorts, ce n'est pas beau là.(...) Ah! C'est frustrant. Mais c'est la seule chose qui est juste sur la terre, on vieillit tous d'un an par année. J'ai eu mon temps. C'est ça que je me dis. (F62)

En outre, I'attribution de la détérioration de l'apparence physique au vieillissement précoce relié au VIH peut rendre ce vieillissement physique encore plus difficile à accepter, comme l'illustrent les propos de cet homme homosexuel.

Sans VIH j'aurais pas vieilli tant que ça dans un coup, ça c'est sûr. Le coup de vieux, ben je l'aurais eu juste à 60 , peut-être, t'sais au lieu de l'avoir à 50, ou ben je l'aurais vu venir progressivement. Tandis que là il est arrivé d'une claque. T'sais comme le gars qui m'a dit: «Tu as vieilli de 10 ans dans une année. ». Il a raison! C'est vrai! (HARSAH53)

Sans être encore une réalité, la crainte d'une dégradation future de la santé en lien avec I'infection à VIH peut parallèlement nuire à l'acceptation de la vieillesse à venir. Les principales craintes formulées par les participants en lien avec le vieillissement futur sont celles de la maladie, de la souffrance et de la perte d'autonomie. Bien que tous les sousgroupes de population soient concernés par ces préoccupations, on peut noter une différence reliée au genre, les participants hommes paraissant plus inquiets de perdre leur autonomie que les femmes.

Plusieurs participants craignent de voir leur santé se dégrader en vieillissant compte tenu de la précarité de leur santé actuelle. Ces craintes peuvent porter sur le risque de l'apparition ou de l'aggravation des comorbidités, sur le risque d'atteindre le stade du sida ou sur la menace d'une dégradation généralisée de l'état de santé. Les propos d'un 
homme hétérosexuel mettent en évidence la distinction établie par les participants entre la vieillesse en elle-même et les risques de maladies qui lui sont associés.

La vieillesse, elle me fait pas peur en tant que telle, c'est la maladie qui fait peur. Parce que n'importe qui, là, si vous me diriez: «Dans tes lignes de la main, là, tu peux vivre 90 ans, pis, euh, ça me dit que tu vas être en santé. » Je vais vivre avec ça! Mais je pense pas que ça soit marqué. (HH65)

La souffrance représente une autre grande source d'inquiétude reliée au vieillissement citée par certains participants. Cette crainte semble d'autant plus prégnante pour ceux ayant assisté à la fin de vie de personnes de leur entourage décédées du sida, comme ce participant: «En vieillissant, ma plus grande peur, c'est la souffrance. Pis en plus, je viens... j’ai vu... La personne en question qui est décédée le 8 décembre, je l'ai quasiment vu mourir là, à petit feu, d'une semaine à l'autre. » (HARSAH62)

Enfin, plusieurs participants hommes et femmes insistent sur leur crainte de ne plus être autonome dans leur vie quotidienne et de dépendre des autres en avançant en âge. La peur de perdre sa mobilité et de devoir se déplacer avec une marchette ou en fauteuil roulant voire d'être totalement immobilisé est apparue particulièrement présente dans les discours des hommes. Pour certains participants, c'est le fait de devoir compter sur les autres pour les soins du corps qui paraît plus préoccupant. D'autres, enfin, mettent davantage l'accent sur la crainte de représenter un fardeau pour leurs proches et de leur imposer de prendre soin d'eux. Un participant explique: «C'est mieux d'arrêter la vie plus tôt possible, que de traîner la vie comme telle. Et surtout les proches de moi, je veux pas qu'eux autres ils me voient comme ça de même. Ça amène beaucoup de problèmes avec les proches qui sont obligés de s'occuper de vous. » (HH51)

Cette crainte de la maladie, de la souffrance ou de la perte d'autonomie conduit certains participants à souhaiter ne pas vivre après un certain âge, qui, selon les représentations de la grande vieillesse de chacun, oscille entre 80 ans et 100 ans. Des répondants confient même songer à mettre fin à leur jour ou espérer pouvoir bénéficier de l'euthanasie si leur santé se dégradait trop ou s'ils arrivaient à un stade de dépendance totale. 
Bien on vient tous au monde pour crever hein! On va tous mourir, mais je vais essayer de ne pas mourir malade, le moins possible. Moi tomber ben malade, je ne le sais pas si je l'endurerais. Moi je pense que je me shooterais une overdose. Bye! T'sais pour devenir impotent, me faire laver par une personne, ou me faire ... Non, non, non. Oublie ça, je ne serais pas capable. (HH65)

Bien que le fait de vivre avec le VIH ne semble pas avoir d'impact sur le vécu présent du vieillissement qui apparaît davantage modelé par l'identité de genre, nos données mettent en évidence les répercussions négatives de I'infection à VIH sur la perception du vieillissement futur de certaines PVVIH50+. Cependant, d'après l'analyse des discours, le fait d'avoir vieilli avec le VIH pourrait, au contraire, avoir une influence positive sur le vécu actuel du vieillissement de plusieurs participants.

\section{- L'acceptation du vieillissement}

La moitié des participants témoigne d'un vécu positif du vieillissement. Dans plusieurs cas, cette acceptation semble prendre sa source dans le parcours relié au VIH. Trois éléments reliés à ce parcours apparaissent jouer un rôle essentiel dans le processus d'acceptation du vieillissement, à savoir les épreuves surmontées, le sentiment d'être un survivant du VIH et l'acceptation de la mort.

Les personnes ayant été infectées dans les premières années de l'épidémie du VIH ont souvent dû faire face à de nombreuses épreuves en lien avec l'infection, qu'il s'agisse de la maladie, d'hospitalisations, de la menace de la mort, de la perte des proches morts du sida ou de la prise des premiers traitements antirétroviraux comportant un grand nombre de pilules et provoquant de multiples effets secondaires. Le fait d'avoir été confronté à ces épreuves marquantes à un jeune âge et de les avoir surmontées après plusieurs années de combat confère une valeur particulière au vieillissement qui devient synonyme de délivrance et de changements positifs. Deux participantes ont fait référence à cette vision positive du vieillissement qu'elles perçoivent avant tout comme une période d'amélioration de leur condition, comme l'illustrent les propos d'une d'entre elles.

Par rapport au vieillissement, moi, j'ai comme une autre approche.

Parce que ce que j'ai vécu avant, c'était comme vide, tu sais? Y a 
eu beaucoup de, c'était difficile avant. Alors maintenant, où j'arrive maintenant, moi, c'est comme... Tu sais, j'ai beaucoup de possibilités, y a encore plein de choses que je veux faire (...) Pour moi, le vieillissement, ça sonne tellement pas comme quelque chose que je veux pas, et que je vois comme quelque chose de terrifiant, parce que je viens de me sortir de quelque chose de difficile, alors moi, y en a pas de problème! (F53)

Dans le même ordre d'idées, le sentiment d'avoir survécu au VIH, c'està-dire d'être encore en vie alors que la mort était attendue depuis de nombreuses années confère au vieillissement une connotation particulièrement positive. Cette situation se retrouve dans le discours de certains participants infectés dans les premières années de l'épidémie, avant l'arrivée des thérapies antirétrovirales hautement efficaces, et pour lesquelles le diagnostic du VIH équivalait à une mort imminente ou relativement proche. Les propos de plusieurs participants, hommes et femmes, démontrent une satisfaction et un étonnement d'avoir atteint l'âge qu'ils ont, ce qui favorise une meilleure acceptation du vieillissement.

Je suis contente parce l'année passée, le 7 juillet, j'ai couru partout, j'ai dit à tout le monde: «Je vais avoir 60 ans, je vais avoir 60 ans! Il y a quelqu'un qui m'a dit: «C'est la première fois que je vois une femme contente parce qu'elle va avoir 60 ans.». Mais les personnes à qui j'ai dit, ils savent pas pourquoi je le dis. Parce que depuis la date que je connais que j'ai cette maladie-là et que je suis toujours là, pour moi c'est quelque chose immense. (F59)

Le dernier élément relié au parcours de vie avec le VIH qui semble avoir une influence sur le vécu positif du vieillissement a trait au rapport à la mort. Bien que les participants n'aient pas établi eux-mêmes un lien entre ces deux phénomènes, I'analyse des données démontre qu'environ deux tiers des participants ayant manifesté leur acceptation du vieillissement évoquent parallèlement leur acceptation de la perspective de la mort ${ }^{4}$. On peut donc supposer que la confrontation à la perspective de la mort à un jeune âge qui caractérise le parcours des PVVIH peut avoir un impact positif sur l'acceptation du vieillissement. L'extrait d'entrevue qui suit permet de mettre en parallèle les propos d'un participant relatifs à son acceptation de la mort et à celle 
du vieillissement, bien qu'ils aient été formulés à des moments différents de l'entrevue.

Je me sens plus éphémère qu'un autre, comme j'ai plus souvent pensé à la mort puis à la maladie, que d'autres, je pense ... Comparé aux gens du même âge que moi...Mais ça commence à les attraper ils se sentent vieillir, ceux de mon âge (rires) (...) Y a-t-il du positif dans le vieillissement? Ben oui, ben oui! Tout est assez positif là. On se prend moins au sérieux, je ris pas mal avec les voisins. C'est agréable... Ça va bien. (HH53)

Pour finir, I'analyse des données met également au jour des variations importantes relatives à l'acceptation du vieillissement en lien avec le genre et I'orientation sexuelle. Alors que les deux tiers des participants hétérosexuels acceptent complètement I'idée de vieillir, c'est le cas de la moitié des participantes et de seulement un tiers des participants HARSAH. Les discours des hommes hétérosexuels dépeignent le vieillissement comme un phénomène normal et attendu. Cette normalisation du vieillissement perçu comme naturel et inévitable, semble largement contribuer à son acceptation, comme l'illustre cette citation.

Le vieillissement, c'est un phénomène normal de la vie, on est jeune, on grandit, on fait beaucoup de choses. Le temps passe et ne se récupère pas. C'est normal. Le corps vieillit physiquement, I'organisme vieillit physiquement aussi et ... Donc c'est un phénomène normal. Ça ne sera aucun problème pour moi (en riant)! ( $\mathrm{HH} 73)$

\section{DISCUSSION ET CONCLUSION}

L'exploration de l'expérience du vieillissement des PVVIH50+ en trois volets, à savoir l'expérience du vieillissement physique, le sentiment de vieillir et le rapport au vieillissement présent et futur, permet de mettre en lumière les spécificités propres à l'expérience de personnes se situant à l'intersection du VIH et du vieillissement mais aussi les similitudes existant entre leur expérience et celle de la population générale âgée. 
Tout d'abord, les PVVIH50+ prennent conscience de leur vieillissement à travers des transformations physiques se situant principalement dans les registres corporels de l'apparence, l'énergie et la santé physique, ce qui recoupe ce qui a été décrit par Vannienwenhove (2009) à propos de la population âgée. La différence majeure réside dans le fait que ces changements surviennent à un âge prématuré, ce qui peut être source de questionnement pour l'individu et rendre leur acceptation plus difficile.

L'analyse des données a par ailleurs montré que la majorité des PVVIH50+ se situe dans une ambivalence au regard du sentiment vieillir, affirmant se sentir encore jeune ou ne pas se sentir vieillir, malgré leur perception de signes physiques de vieillissement. Ces résultats font également écho à ceux d'autres travaux qui insistent sur la volonté des aînés de ne pas se voir assigner une identité stigmatisée de personne âgée. Les auteurs observent la distinction établie par les personnes âgées entre le processus de vieillissement et l'état de vieillesse ou «identité de vieux» (Balard, 2011) ou bien entre le «devenir vieux» et I'«être vieux» (Caradec, 2008), le second positionnement faisant généralement figure de repoussoir, bien que les personnes entrant dans la grande vieillesse l'assument plus facilement. Les PVVIH50+ cherchent eux aussi à se distancier de I' «identité de vieux», qui pourrait leur être assignée au regard de leur vieillissement physique, en dépit de leur âge chronologique encore peu avancé. Ce travail de distanciation s'opère généralement à travers trois principales stratégies, à savoir la relativisation des signes physiques de vieillissement par ceux d'un maintien de la jeunesse, le regard des autres et la comparaison entre soi et les autres, ce qui recoupe les pratiques mises en évidence par Vannienwenhove (2009) au sujet de la population âgée générale.

En ce qui concerne le sentiment d'être vieux, il peut naître chez les PVVIH50+ d'une dégradation de la santé qui empêche d'accomplir des activités réalisées tout au long de l'existence, comme décrit dans la littérature sur les populations âgées (Caradec, 2008; Balard, 2011), à la seule différence que ce sentiment se développe à un âge plus jeune chez les PVVIH50+. Cependant, si la perte des aptitudes physiques constitue, pour la majorité des participants, le déclencheur du sentiment $d^{\prime}$ «être vieux», ce sentiment peut aussi s'enraciner dans la dégradation de l'apparence qui porte atteinte à l'image de soi et entraîne une rupture dans les sphères intime et sociale de la trajectoire 
biographique. Quoiqu'il en soit, dans les deux cas de figure, le sentiment d'être vieux trouve son origine dans l'impression d'une rupture entre ce que les personnes ont été et ce qu'elles sont, ce qui rejoint les analyses de Caradec (2008).

La problématique de l'acceptation du vieillissement présent et à venir par les PVVIH50+ s'est avérée à la fois teintée des normes relatives au genre et du parcours relié au VIH. Corroborant les résultats de nombreux travaux, notre recherche fait ressortir l'expérience différentielle du vieillissement en fonction du genre. Le vécu plus difficile du vieillissement présent chez les PVVIH50+ étant principalement relié à la difficile acceptation de la transformation de l'apparence physique, il est peu surprenant qu'il touche davantage les HARSAH et les femmes. La littérature sur l'expérience du vieillissement souligne en effet le double standard existant entre les hommes (hétérosexuels) et les femmes en matière de vieillissement, ces dernières devant faire face simultanément à l'âgisme et au sexisme. Comme le souligne Sontag (1978, citée par Featherstone et Wernick, 1995), il est attendu des femmes qu'elles se conforment à l'idéal adolescent tout au long de leur vie, la présentation corporelle étant au cœur de l'identité féminine. L'apparence physique représente, par conséquent, un enjeu central dans l'expérience des femmes vieillissantes et âgées, qui se voient dénier le droit de vieillir physiquement et souffrent de ne pouvoir se conformer aux standards sociétaux de beauté axés sur la jeunesse (Hurd, 1999; Hurd Clark et Korotchenko, 2011; Krakula, 2007). La transformation de I'apparence serait beaucoup moins stigmatisée et source de discrimination pour les hommes (hétérosexuels) que pour les femmes (Ward et Holland, 2011), I'identité masculine se fondant davantage sur les aptitudes physiques et la force (Hurd Clark et Korotchenko, 2011). De même les recherches plus rares sur l'expérience du vieillissement des hommes gais pointent la valeur fondamentale accordée au corps et à la préservation d'une apparence jeune, musclée et belle, au sein de la communauté gaie, qui rend l'expérience du vieillissement particulièrement difficile à vivre dans ce groupe. (Slevin et Linneman, 2009 ; Hurd Clark et Korotchenko, 2011).

En revanche, il est apparu que c'était la perte d'autonomie physique risquant de survenir avec l'avancement en âge qui représentait la plus grande inquiétude pour les participants hommes hétérosexuels. Ce résultat confirme ceux d'autres travaux qui pointent le vécu négatif du 
déclin fonctionnel des hommes hétérosexuels âgés dans la mesure où la force et l'indépendance sont étroitement reliées à l'identité masculine (Hurd Clarke et Korotchenko, 2011).

Si l'expérience des PVVIH50+ s'accorde donc parfaitement avec celle des femmes et des hommes (hétérosexuels et homosexuels) âgés de la population générale concernant le vécu négatif du vieillissement, elle en diffère cependant par la vision positive du vieillissement dont témoigne la moitié des participants. Alors que la population générale des baby-boomers (génération dont font partie la majorité des participants à notre recherche) tend à percevoir le vieillissement comme un phénomène négatif en raison de son association avec la maladie et la mort (Blein et al., 2009), une partie des PVVIH50+ le considère au contraire comme un horizon riche de multiples possibilités et un immense privilège. Si le fait d'avoir survécu aux innombrables épreuves associées au parcours le VIH contribue grandement à cette vision positive du vieillissement, le cheminement effectué précocement par les PVVIH50+ par rapport à la problématique de la finitude humaine pourrait aussi être une des pierres angulaires de cette acceptation du vieillissement. Des recherches ont en effet montré le lien existant entre la peur de vieillir et celle de la mort chez les baby-boomers (Blein et al., 2009), et à l'inverse, entre la vieillesse et la conscience de la finitude ou l'acceptation de la mort à venir (Heikinnen, 2000 et 2004; Hummel 2001). Avoir été confronté à un jeune âge à la menace de la mort pourrait largement expliquer le rapport positif au vieillissement que nourrit une proportion importante de PVVIH50+. Bien que la perspective future de vieillir avec le VIH revête un caractère inquiétant pour les participants, en raison des risques prononcés de dégradation de leur santé, il semble que ce soit moins la vieillesse qui soit redoutée en elle-même que les troubles graves de santé et la perte d'autonomie qui risquent de l'accompagner. Alors que les baby-boomers ont peur de vieillir car ils associent la vieillesse à la maladie, dans le cas des PVIH50+, qui ont déjà pour beaucoup expérimenté la maladie, c'est bien la peur de tomber encore plus gravement malade qui les effraie et non le vieillissement en lui-même.

Pour conclure, bien qu'un certain nombre de PVVIH50+ souhaitent se distancier du vieillissement, témoignant de leur internalisation de l'âgisme qui prédomine dans les sociétés occidentales, d'autres se démarquent par un vécu positif du vieillissement qui s'enracine dans 
un parcours de lutte contre une maladie autrefois mortelle ayant fait naître en eux un sentiment d'être chanceux d'avoir survécu et de pouvoir vieillir (voir Wallach, 2011). À la lumière des résultats de cette recherche, le parcours avec le VIH qui pourrait au premier abord être envisagé comme une source de vulnérabilité, se révèle donc, au fil des discours, comme une force et une ressource majeure pour faire face à l'expérience du vieillissement.

\section{BIBLIOGRAPHIE}

BALARD, F. (2011). «Vivre et dire la vieillesse à plus de 90 ans, se sentir vieillir mais ne pas être vieux», Gérontologie et société, vol. 3, n 138, p. 231-244.

BLEIN, L., J.-P. LAVOIE, N. GUBERMAN et J.I. OLAZABAL (2009). «Vieillirontils un jour? Les baby-boomers aidants face à leur vieillissement», Lien social et Politiques, vol. 62, p. 123-134.

BRENNAN, M., S.E. KARPIAK, R.A. SHIPPY et M.H. CANTOR (dir.), (2009). Older adults with HIV, New York, Nova Science Publishers.

CARADEC, V. (2008). Sociologie de la vieillesse et du vieillissement, Barcelone, Armand Colin.

DENZIN, N.K. et Y. LINCOLN (1994). «Introduction: Entering the field of qualitative research», dans N.K. DENZIN et Y. LINCOLN (dir.), Handbook of qualitative research, Thousand Oaks, Sage, p. 1-17.

DESQUILBET, L., L.P. JACOBSON, L.P. FRIED, J.P. PHAIR, B.D. JAMIESON, M. HOLLOWAY, J.B. MARGOLICK et MULTICENTER AIDS COHORT STUDY (2007). «HIV-1 infection is associated with an earlier occurrence of a phenotype related to frailty», Journal of Gerontology Series A: Biological Sciences and Medical Sciences, vol. 62, n० 11, p. 1279-1286.

EFFROS, R.B., C.V. FLECHTER, K. GEBO, J.B. HALTER, W.R. HAZZARD, F.M. HORNE et R.E. HUEBNER (2008). «Aging and infectious diseases: workshop on HIV infection and aging: what is known and future research directions», Clinical Infectious Disease, vol. 47, n 4, p. 542-553.

EMLET, C.A. (dir.), (2004). HIV/AIDS and older adults, New York, Springer Publishing Company.

EMLET, C. A., S. TOZAY et V. H. RAVEIS (2010). «"I'm not going to die from the AIDS": Resilience in aging with HIV disease», The Gerontologist, vol. 51, $\mathrm{n}^{\circ} 1$, p.101-111.

FEATHERSTONE, M. et A. WERNICK (dir.), (1995). Images of aging: Cultural representation of later life, London et New York, Routledge.

HEIKKINEN, R.-L. (2000). «Ageing in an autobiographical context», Ageing and Society, vol. 20, p. 467-483.

HEIKKINEN, R.-L. (2004). «The experience of ageing and advanced old age: a ten-year follow up», Ageing and Society, vol. 20, p. 567-582

HUMMEL, C. (2001). «Représentations de la vieillesse chez des jeunes adultes et des octogénaire», Gérontologie et société, vol. 3, n 98, p. 239-252. 
HURD, L.C. (1999). «"We're not old!": Older women's negotiation of aging and oldness», Journal of aging studies, vol. 13, $\mathrm{n}^{\circ} 4$, p. 419-439.

HURD CLARKE, L. H. et A. KOROTCHENKO (2011). «Aging and the body: A review», Canadian Journal on Aging, vol. 30, n 4, p. 495-510.

KATZ, S. (2009). Cultural aging. Life course, lifestyle, and senior worlds, Toronto, University of Toronto Press.

KREKULA, C. (2007). «The intersection of aging and gender: Reworking gender theory and social gerontology», Current Sociology, vol. 55, $\mathrm{n}^{\circ} 2$, p. $155-171$.

LAGACÉ, M. (2010). «Introduction», dans M. LAGACÉ (dir.), L'âgisme. Comprendre et changer le regard social sur le vieillissement, Québec, Presses de I'Université Laval, p. 1-9.

POWER, L., M. BELL et I. FREEMANTLE (2010). A national study of aging and HIV (50 plus), York, Joseph Rowntree Foundation.

SLEVIN, K.F. (2008). «Disciplining bodies: The aging experience of older heterosexuel and gay men», Generations, p. 36-42.

SLEVIN, K. F. et T. J. LINNEMAN (2010). «Old gay men's bodies and masculinities », Men and Masculinities, vol. 12, n 4, p.483-507.

STRAUSS, A. et J. CORBIN (1998). Basic of Qualitative Research (2e éd.), California, Sage Publications.

VANNIENWENHOVE, T. (2009). «La corporéité de I'individu vieillissant. Entre images collectives et expériences individuelles», dans OLAZABAL, I. (dir.), Que sont les baby-boomers devenus? Aspects sociaux d'une génération vieillissante, Cap-Saint-Ignace, Éditions Nota Bene, p. 109-135.

WARD, R. et C. HOLLAND (2010). " "If I look old, I will be treated old": Hair and later-life image dilemmas», Ageing \& Society, vol. 31, p. 288-307.

WALLACH, I. (2011). ""Je suis heureux d'avoir l'âge que j'ai": la résilience des hommes gais âgés vivant avec le VIH au Québec», Revue canadienne de santé mentale communautaire, vol. 30, n² 2, p. 157-171.

\section{NOTES}

1 Ce vieillissement serait dû à l'affaiblissement du système immunitaire et I'inflammation continue de l'organisme causé par le VIH ainsi qu'à l'exposition au long cours aux effets toxiques des médicaments antirétroviraux.

2 Le VIH est passé du statut de maladie mortelle à celle de maladie chronique grâce à l'avènement des thérapies antirétrovirales hautement efficaces en 1996.

3 Les codes mentionnés après une citation visent à donner une information sur le genre, l'orientation sexuelle et l'âge du participant au moment de I'entrevue. Ainsi HH60 signifie homme hétérosexuel de 60 ans. $\mathrm{F}$ correspond à femme et HARSAH à homme ayant des rapports sexuels avec des hommes.

4 Nous n'incluons pas ici les personnes ayant mentionné le désir de mourir ou de mettre fin à leurs jours en cas de dégradation de leur santé, ce qui peut être perçu comme un refus du vieillissement (voir Blein et al., 2009) 